\title{
Numerical Investigation of Surface Acoustic Wave (SAW) Interacting with a Droplet for Point-of-Care Devices
}

\author{
Imran Shah \\ Department of Mechatronics Engineering, Jeju National University, Jeju, Republic of Korea. \\ School of Mechanical $\mathcal{E}$ Manufacturing Engineering, National University of Sciences and Technology H-12, Is- \\ lamabad, Pakistan.

\begin{abstract}
Emad Uddin, Aamir Mubashar and Muhammad Sajid
School of Mechanical E Manufacturing Engineering, National University of Sciences and Technology H-12, Islamabad, Pakistan.
\end{abstract}

\author{
Muhammad Yamin Younis \\ Department of Mechanical Engineering, Mirpur University of Science and Technology (MUST), Mirpur 10250 \\ (AJK), Pakistan.
}

\begin{abstract}
Hudair Samad
School of Mechanical E Manufacturing Engineering, National University of Sciences and Technology H-12, Islamabad, Pakistan.
\end{abstract}

\author{
Kyung Hyun choi \\ Department of Mechatronics Engineering, Jeju National University, Jeju, Republic of Korea.
}

\begin{abstract}
(Received 17 November 2016; accepted 18 February 2019)
A three-dimensional numerical simulation of the interaction of a surface acoustic wave (SAW) with a droplet of water is carried out. The mixing produced inside the droplet due to the incident with the SAW and the droplet is investigated by undertaking a parametric study, with parameters such as frequency, drop size, and the lateral position of the droplet on the surface of the substrate. The linear relationship between the input voltage and the mixing velocity inside the droplet is obtained with variation of the input voltage of the inter-digital transducer (IDT) of the SAW device within a 10-40 V range. With the variation in frequency, the maximum mixing velocity is observed at $20 \mathrm{MHz}$ and it appears to be independent of the size of the droplet. Varying the substrate material with lead zirconate titanate and lithium niobate produces better mixing. Lithium niobate is preferred due to its availability and cost-effectiveness. A drop of $600 \mu \mathrm{m}$ diameter produces better mixing. The different velocities inside the drop and the SAW device are obtained by changing the droplet position in the lateral direction (asymmetrical position) from the centre of the substrate. Cut planes parallel and perpendicular to the SAW at the core of a half-spherical droplet are observed to visualise the mixing effects inside the droplet during the interaction. To achieve the best mixing criteria, the droplet is moved in a lateral direction. An efficient parametric design for the mixing phenomena in micro-fluidic devices is presented for point-of-care devices.
\end{abstract}

\section{INTRODUCTION}

Micro-fluidics and micro-scale tools are the emerging technologies in the field of life sciences, and droplet micro-fluidics is proving itself to be a good substitute to continuous microfluidics, especially in biochemical analysis. Nonetheless, micro-droplet technology has opened a new podium for microscaled chemical reactions, but it still needs effective actuation of individual droplets at microscopic scales. ${ }^{1}$ However, it is complicated to achieve mixing inside a droplet at a very low Reynolds number and at a microscopic scale. ${ }^{2}$ A surface acoustic wave (SAW) device working on the principal of surface acoustic waves can handle the problem of mixing pattern propagation inside a droplet in an improved manner. ${ }^{3}$ An SAW could be produced by giving an input voltage to the interdigital transducer (IDT), on the facade of the substrate of an SAW device, as shown in Fig. 1. When a water drop is positioned in the path of a SAW, the wave becomes a leaky surface acoustic wave (LSAW). After the interaction with the droplet, owing to the variation in the wave speed in the droplet and the solid substrate, the wave propagated with the Rayleigh angle $\Theta_{R}$, as shown in Fig. 2. ${ }^{4}$ Mathematically, $\Theta_{R}=\sin ^{-1}\left(C_{l} / C_{s}\right)$, where $C_{1}$ and $C_{s}$ were the speed of sound in liquid and substrate material, respectively, and its value was approximately $23^{0}$, in the case of the water droplet. ${ }^{5}$ The penetration depth of the Rayleigh SAW inside the substrate was inversely proportional to the applied frequency. ${ }^{6}$

The SAW device played a vital role in the generation of acoustic mixing, which, in turn, facilitated pumping, ${ }^{7,8}$ stirring, ${ }^{9,10}$ concentration of particles, ${ }^{11}$ vibrating, ${ }^{12,25}$ and atomization. $^{13,14}$ Acoustic mixing inside a droplet due to a SAW interaction is a three-dimensional phenomenon, but to reduce the computational time and complications in geometry modelling, a past two-dimensional analysis was performed to study this phenomenon. ${ }^{15}$ For the same reason, there are very few re- 


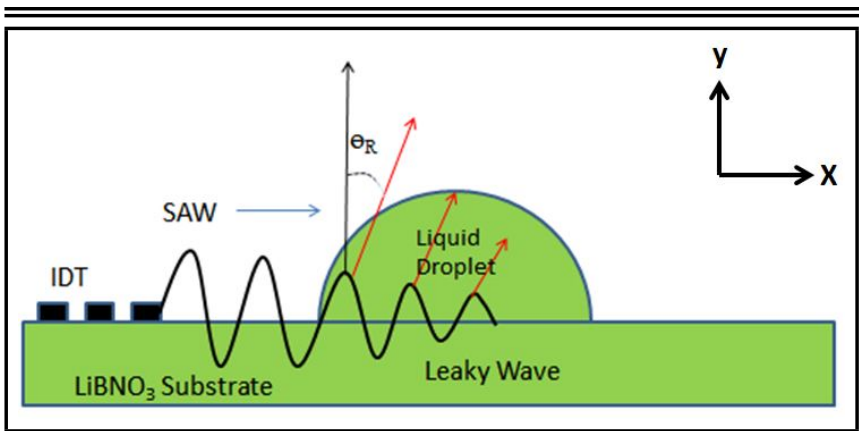

Figure 1. 2-D scheme of leak wave propagation inside droplet with $\Theta_{R}$.

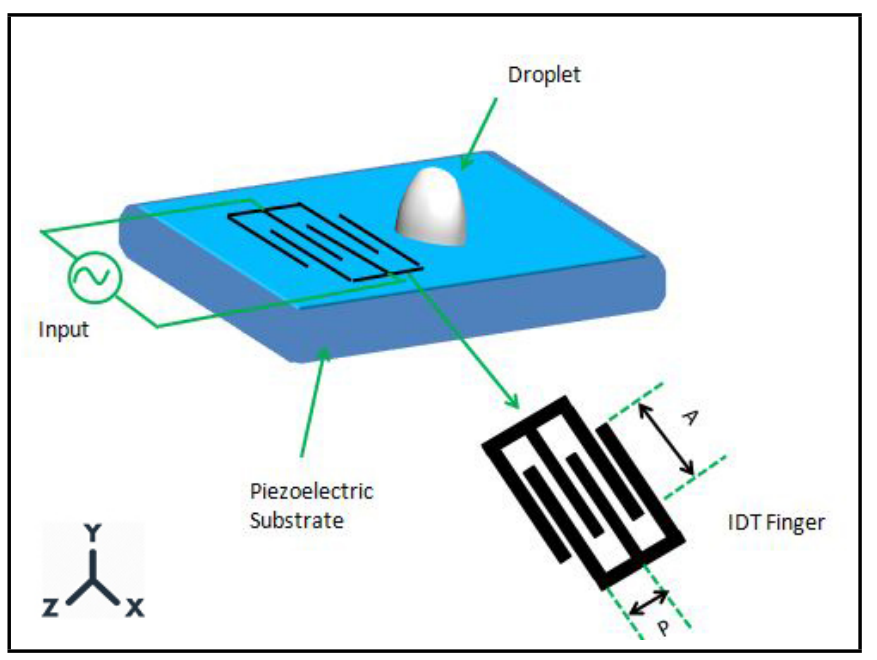

Figure 2. 3-D schematic of SAW propagation from IDT, that interacts with the droplet on the surface of the substrate.

ported cases which extend up to the 3-D model design of efficient SAW devices for micro-fluidics and lab-on-chip devices. ${ }^{5}$ Despite it being a complete understanding of the mixing affects inside a droplet of water, it can only be undertaken by a complete three-dimensional analysis.

Frommelt et al. introduced a micro pump based on the SAWs. ${ }^{16}$ They conducted both a simulation and an experimentation, and concluded that the use of an SAW device is not limited to mixing and pumping, but rather it can also be utilized in the "lab-on-chip devices" aimed towards biological and pointof-care devices. ${ }^{16}$ Luo et al. studied the effect of droplet position on the surface of a substrate using two simulated cases. ${ }^{17}$ The first case dealt with a droplet of a size less than the IDT aperture placed in the path of the SAW, and in the second one, the droplet position was changed, keeping it partially in the path of the SAW. They reported completely different flow patterns for both cases, as the flow pattern was symmetric for the first case, but turned asymmetric in the second. The streaming velocity at different RF powers was applied to a SAW and they observed a direct relationship between the applied RF powers and the streaming velocity. ${ }^{17}$

This paper presents the 3-D numerical investigation of a SAW micro droplet and the flow produced inside the droplet, as result of SAW interaction, including the effects of frequency, lateral drop position, input voltage, and substrate material. As a detailed study undertaking these parameters collectively in detail is unavailable, the results would provide vital information for the selection of input voltage, applied frequency, lateral position of droplet on substrate, and better material usage for the substrate of a SAW device. This parametric study will be a benefit for micro-fluidic and lab-on-chip devices recently having great role in point-of-care and heath recovery devices.

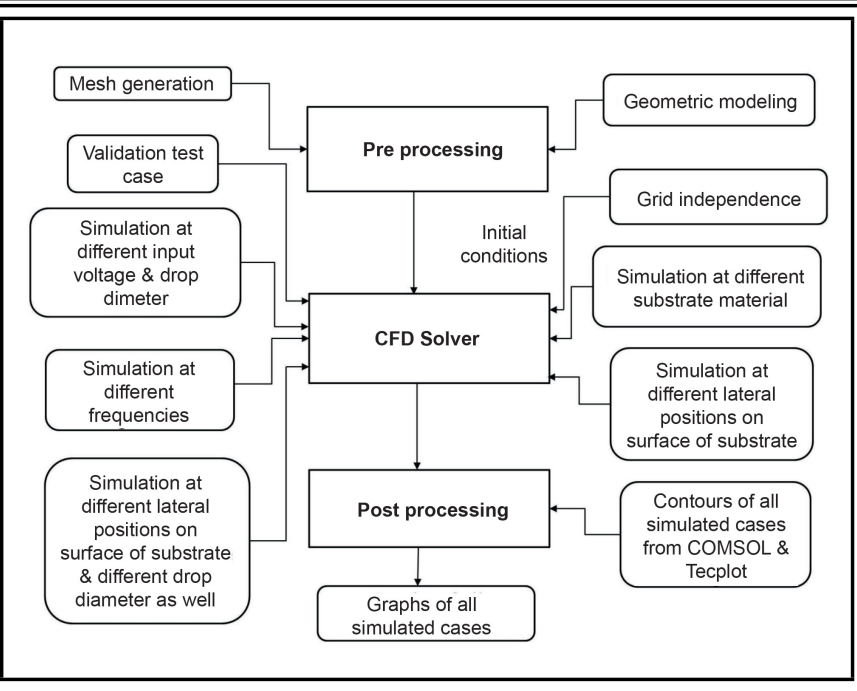

Figure 3. Flow chart of numerical modelling of the problem.

Section 2 of this document explains the numerical methodology, Section 3 provides a detailed discussion of the obtained results, and Section 4 provides the conclusions drawn from this work.

\section{NUMERICAL ANALYSIS}

\subsection{Numerical Model}

This study considered the liquid as a Newtonian fluid defined by its density, characteristic speed of sound, and viscosity. The flow chart of the whole process is provided in Fig. 3. The simulation domain covered the contact of the liquid and the piezoelectric surfaces, connected to each other via appropriate boundary conditions, and a no-slip condition with the liquid interface was also assumed. The interface between the piezoelectric-air domains and liquid-air domains was considered as a soft boundary for the acoustic waves where the pressure is zero.

A SAW liquid coupling model was developed using COMSOL CFD module (COMSOL Inc.). Owing to the hydrodynamic behaviour of the droplet, obtained as a result of SAW interaction with it, the droplet of water was assumed to be governed by a laminar incompressible Navier-Stokes continuity and the momentum equation driven by some external force, F, generated due to the interaction of the SAW with the droplet of liquid.

$$
\begin{gathered}
\overrightarrow{\nabla \cdot U}=0, \\
\frac{\partial U}{\partial t}+(U \cdot \vec{\nabla}) U=F-\overrightarrow{\nabla p}+v \cdot \overrightarrow{\nabla^{2}} U .
\end{gathered}
$$

In the stated equations, $U$ represented the acoustic wave streaming velocity, $p$ was the kinematic pressure and $v$ was the fluid kinematic viscosity. The calculation of acoustic streaming motion was forced by the external body force $F$ in the momentum equation. This force was caused by a spatial gradient of Reynold stress of fluctuating flow due propagation of the sound wave within the fluid medium as stated by the Lighthill theory. However, the solutions of Eq. (1) and Eq. (2) required knowledge of SAW force distribution, $F$.

According to the theory of acoustic streaming, $F$ could be calculated as:

$$
F=-\left(1+\alpha_{1}^{2}\right)^{\frac{3}{2}} A^{2} \omega^{2} k_{i} \exp 2\left(k_{i} x+\alpha_{1} k_{i} z\right),
$$




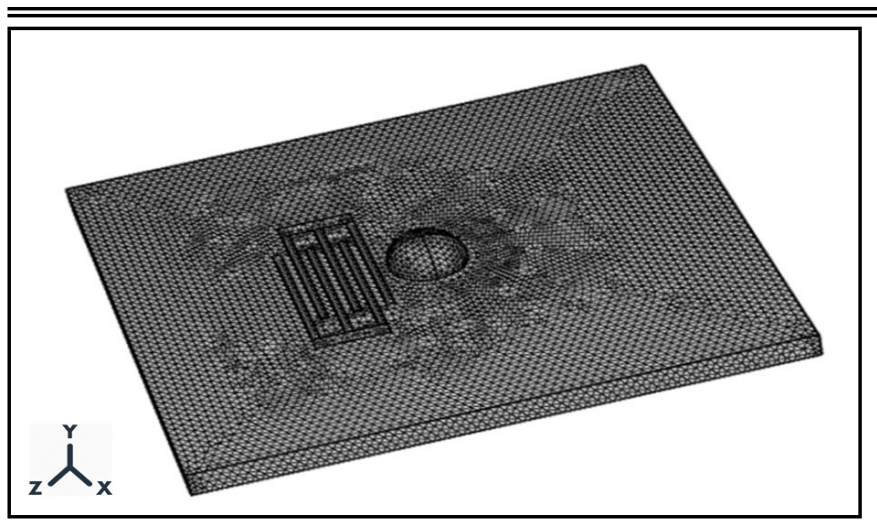

Figure 4. Optimal meshing geometry of the problem.

where $\omega$ was the angular frequency and $A$ was representing the amplitude of the SAW at a point of interaction between the SAW and the liquid droplet. A leaky SAW wave number denoted by $K_{L}$ was given by the formula:

$$
K_{L}=K_{r}+J K_{i}
$$

For the case of solid-liquid coupling, the wave number could be obtained by applying the Campbell and Jones method. The attenuation constant in the above equation, (C) was $\alpha_{1}=J \alpha$, and $\alpha=1-\left(\frac{C_{L}}{C_{W}}\right)^{2}$, where $C_{L}$ and $C_{W}$ were the leaky SAW velocity and the velocity of sound in a liquid, respectively. The wave propagates inside the droplet with an angle, named the Rayleigh angle, which could be calculated as:

$$
\theta_{R}=\sin ^{-1}\left(\frac{C_{W}}{C_{S}}\right)
$$

The total force of streaming $F$ can be expressed by formula, $F=\left(F^{2} x+F^{2} y\right)^{1 / 2}$, where it was independent of the $y$-direction. This study restricted the value of the applied voltage to the IDT up to a certain limit to avoid droplet deformation and movement due to interaction with the SAW.

\subsection{Meshing and Solution}

A grid independence study was carried out after the creation of a geometric model for the problem and for this purpose the mesh size was varied from extremely coarse to fine until the solution became independent of the mesh size. Figure 4 presents the optimum mesh attained for this study.

A total of sixteen simulations were carried out, varying the mesh size until the problem became grid independent. The number of cells versus the velocity inside the droplet is plotted in the Fig. 5. It was evident through Fig. 5 that the problem was grid independent for all average, maximum, and minimum values. It should be noted that the grid-independent plot shows only the results of the five final simulations. Henceforward, all simulations were performed according to the design of the experiment listed in Table 1. For the solution, the "Time Dependent, Generalized Alpha Method" was used. After 146,829 cells in the domain, the average, minimum, and maximum velocity have negligible change in their values, thus the grid with 146,829 cells was fixed for further simulations.

\subsection{Design of Experiment Table for Simulation}

Table 1 lists the design of the experiment for the further simulations.

As shown in the Fig. 1, $A=1665 \mu \mathrm{m}$ was the aperture of the SAW device and $p=333 \mu \mathrm{m}$ was the pitch of IDT. From

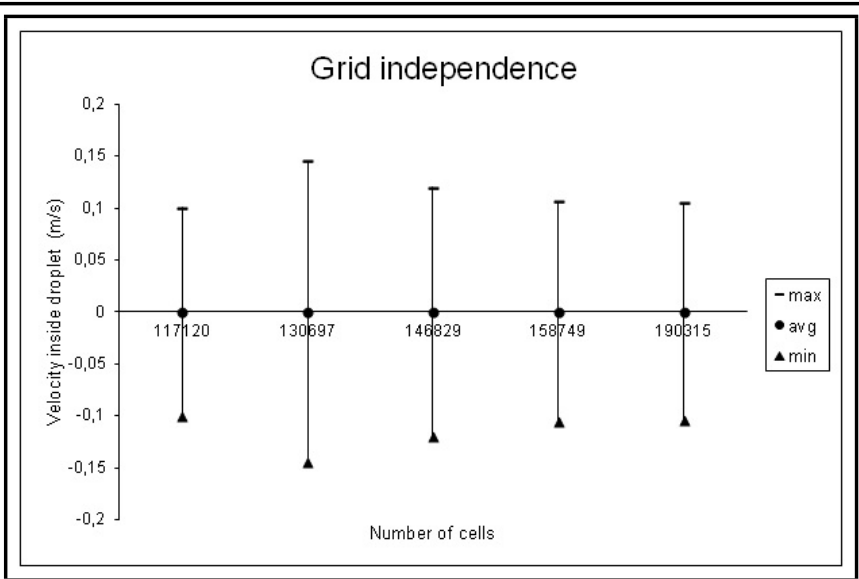

Figure 5. Grid independence plots in which number of cells is plotted against the minimum, average, and maximum velocity inside the droplet, showing clearly that the problem is grid independent.

Table 1. Showing the design of the experiments for the simulations at different parameters for finding the efficient parametric values for mixing in "lab-onchip" and "microfluidic devices" at the micro scale.

\begin{tabular}{||c|c|c|c|c|c||}
\hline $\begin{array}{c}\text { Case } \\
\text { Number }\end{array}$ & $\begin{array}{c}\text { Frequency } \\
(\mathrm{MHz})\end{array}$ & $\begin{array}{c}\text { Lateral } \\
\text { position } \\
(\mu \mathrm{m})\end{array}$ & $\begin{array}{c}\text { Voltage } \\
(\mathrm{V})\end{array}$ & $\begin{array}{c}\text { Substrate } \\
\text { Material }\end{array}$ & $\begin{array}{c}\text { Drop } \\
\text { size } \\
(\mu \mathrm{m})\end{array}$ \\
\hline Case \# 1 & 20 & 0 & $10-40$ & $\begin{array}{c}\text { Lithium } \\
\text { Niobate }\end{array}$ & 600 \\
\hline Case \# 2 & $20-80$ & 0 & 10 & $\begin{array}{c}\text { Lithium } \\
\text { Niobate }\end{array}$ & 600 \\
\hline Case \# 3 & 20 & 0 & 10 & $\begin{array}{c}\text { Lithium } \\
\text { Niobate, } \\
\text { Quartz, } \\
\text { lithium } \\
\text { tanalate, } \\
\& \text { lead } \\
\text { Zirconate } \\
\text { titanate }\end{array}$ & 600 \\
\hline Case \# 4 & 20 & $0-1000$ & 10 & $\begin{array}{c}\text { Lithium } \\
\text { Niobate, }\end{array}$ & 600 \\
\hline Case \# 5 & 20 & $100-700$ & 10 & $\begin{array}{c}\text { Lithium } \\
\text { Niobate, }\end{array}$ & $600-800$ \\
\hline
\end{tabular}

the literature, as well as our frequency effect plot as shown in Fig. 7, it was observed that the maximum mixing results could be obtained at $20 \mathrm{MHz}$, so it was kept constant for the whole of the numerical analysis. ${ }^{9,18}$ Also the value of the SAW device velocity was kept constant at $3996 \mathrm{~m} / \mathrm{s}$ for all simulations. ${ }^{19}$

\section{NUMERICAL RESULTS AND DISCUSSION}

\subsection{Validation}

For validation of the test case results, the input voltage to the IDT was changed and plotted against different droplet sizes, as presented in Fig. 6. A linear relationship was obtained between the input voltage and the maximum velocity. The same trend was reported by Renaudin et al. and Imai et al., showing linear proportionality between the signal voltage versus the velocity. ${ }^{20,21}$ As this study was focused on the inside velocity of the droplet without a significant alteration in shape and with no deformation, the increase in input voltage above $40 \mathrm{~V}$ lead to the complete distortion of the droplet shape, hence the maximum voltage limit, which lead to the droplet deformation that was defined for the given parameters.

As shown in Fig. 6, an improved mixing effect was observed with the droplet of a smaller diameter. Also, with the increase in the input voltage, the maximum velocity of mixing inside the droplet also increased. This could be explained due to the 


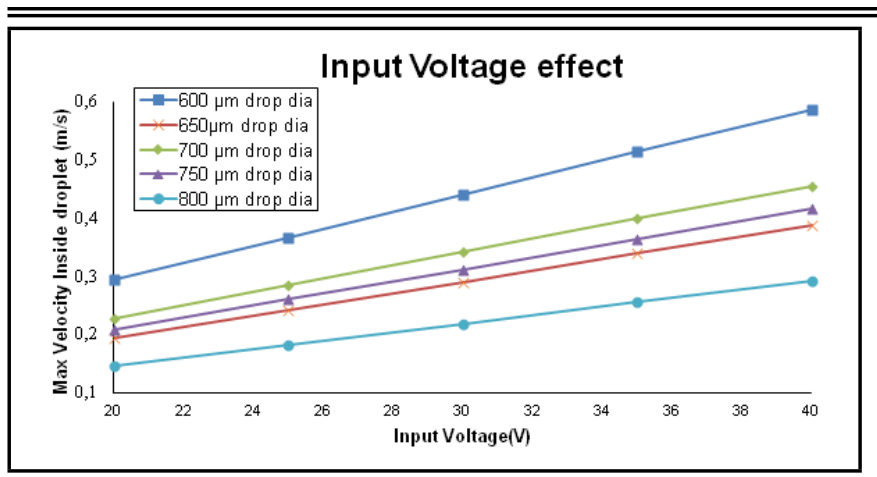

Figure 6. Shows maximum velocities inside the droplet, when device interacted with a very small amount of water.

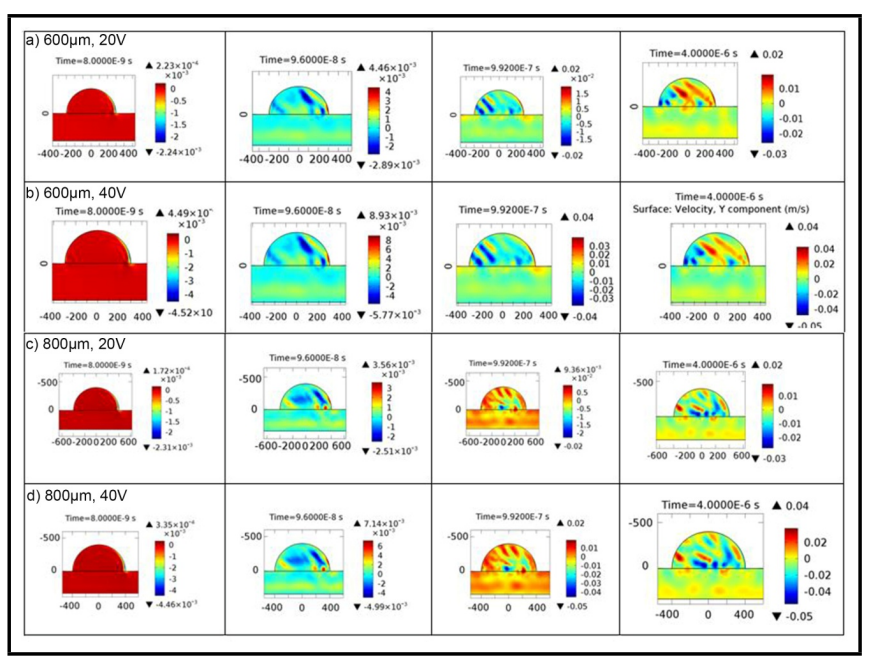

Figure 7. $600 \mu \mathrm{m}$ diameter at $20 \mathrm{~V}, 40 \mathrm{~V} \& 800 \mu \mathrm{m}$ diameter at $20 \mathrm{~V}, 40 \mathrm{~V}$.

increase in the intensity of the wave, which was directly proportional to the voltage, i.e. increasing the voltage of the IDT resulted in an intense wave. Moreover, the intensity was also in direct relationship with the velocity, so with the increase in the input voltage, the velocity inside the droplet also increased.

Figure 7 provides the velocity contours of the droplet. Rows "a" and "b" provide the cut plane from centre of a $600 \mu \mathrm{m}$ diameter droplet. The droplet had different result at different times of simulation, which implied that the velocity inside the droplet was changing with the passage of time. The rows with an increased input voltage of the IDT had an increased velocity inside the droplet. A similar trend can be observed in the rows "c" and "d" in the case of an $800 \mu$ m diameter droplet. Hence, we could conclude from this contour that a higher input IDT voltage provided better mixing.

\subsection{Effect of Frequency}

The streaming effect caused by the variation in frequency was simulated at four different frequencies according to the design of the experiment as presented in Table 1. The graph obtained as a result of the simulated experiments is presented in Fig. 8.

Figure 9 shows that a better streaming/mixing effect inside the droplet was produced with $20 \mathrm{MHz}$ frequency. As the velocity obtained was almost constant at $3996 \mathrm{~m} / \mathrm{s}$, so by equation $v=f \lambda$, the product will remain the same and the frequency will be inversely proportional to the wavelength. Hence, a low frequency will provide a higher wavelength, resulting in a better velocity or streaming effect inside the droplet.

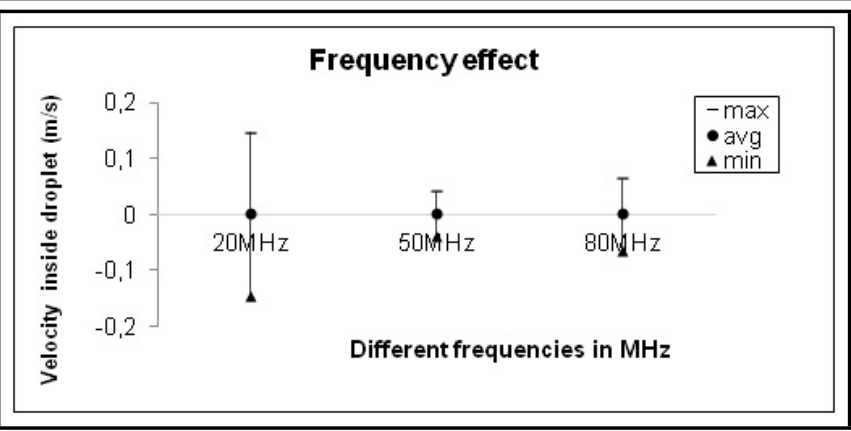

Figure 8. Frequency effect plot shows that the simulations were carried out at different frequencies and it was observed that effective mixing occurred in the case of $20 \mathrm{MHZ}$.

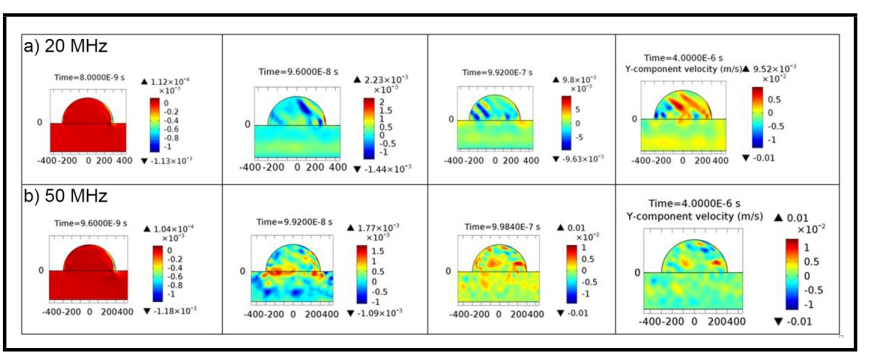

Figure 9. Contour for $20 \& 50 \mathrm{MHZ}$ frequency respectively row wise, with the passage of time in simulation there is a definite change in velocity inside the droplet, increasing.

Streaming could not be attained at the frequency of $50 \mathrm{MHz}$, instead this frequency resulted in a turbulent flow. The literature also designates $20 \mathrm{MHz}$ as the optimum frequency for better mixing for any sized droplet, so we limited our experiments to this frequency. ${ }^{23}$

\subsection{Effect of substrate material}

Four different piezoelectric substrate materials were used namely lithium niobate, lead zirconate titanate, lithim tantalite, and quartz. Better streaming effects were observed in the cases of lead zirconate titanate \& lithium niobite, as shown in the Fig. 10. This effect could be explained by the high charge sensitivity of lead zirconate \& lithium niobite, which caused more inside-droplet velocity for all minimum, average, and maximum values. Results were also dependent on the intermolecular bonding of the substrate material as it is different for each substrate material. ${ }^{24}$

The contours for the cut plane perpendicular to the SAW di-

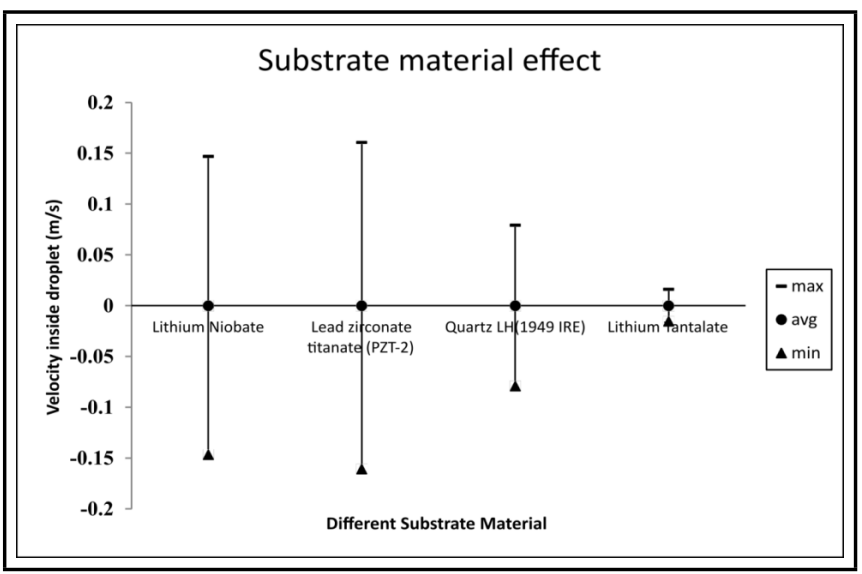

Figure 10. Showing the results of different substrate materials, it was observed that an efficient and better mixing occurred in the case of using lead zirconate titanate (PZT-2) as a substrate material for the SAW device. 


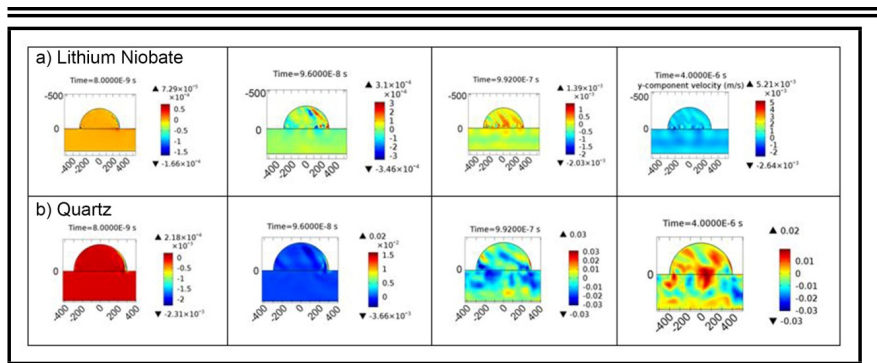

Figure 11. Contours for lithium niobate and quartz as a substrate material, respectively, in row order at different times during the simulations.

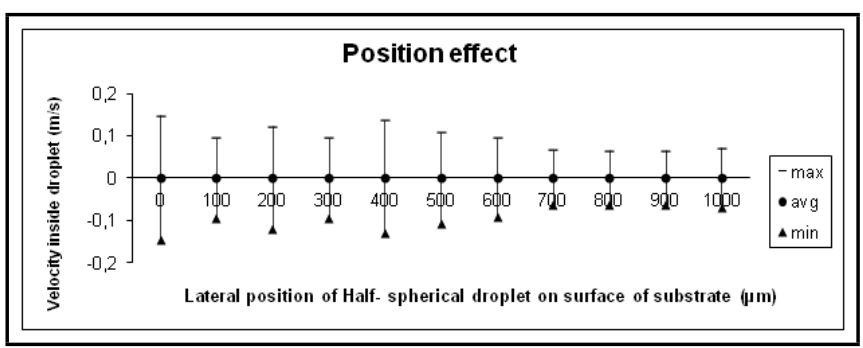

Figure 12. Lateral position of the droplet on the surface of substrate effect, it was observed that up to $600 \mu \mathrm{m}$ diameter of droplet, the trend of mixing is alternatively changing while after $600 \mu \mathrm{m}$, it remained the same for all onward lateral positions of the droplet on the surface of substrate.

rection for lithium niobate having the maximum inside-droplet velocity, and also for the quartz substrate material having less inside-droplet velocity at a different time of simulation are shown in Fig. 11.

\subsection{Effect of Lateral Position}

The droplet was moved from its initially centred position in the lateral direction on the surface of the substrate. It is clear from the Fig. 12 that better streaming was produced when the droplet was at 0 , i.e. at the centre of substrate. Also, the results were alternatively changing its streaming pattern with a change in position, but after the 700 micro-meter position, the effect of streaming became constant through the remaining positions, as it experienced less exposure to the SAW.

Cut plane results for the effect of the lateral position presented in Fig. 13 shows that the centre position produced some streaming velocity at $500 \mu \mathrm{m}$ and the streaming effects were diminished at a distance of $1000 \mu \mathrm{m}$.

\subsection{Effect of Changing Size and Lateral Position}

In this case, both the droplet size and the lateral position of the droplet were changed. The average velocity contours are shown in Fig. 14, by changing the size and the lateral position of the droplet. The surface map provided the optimal ranges

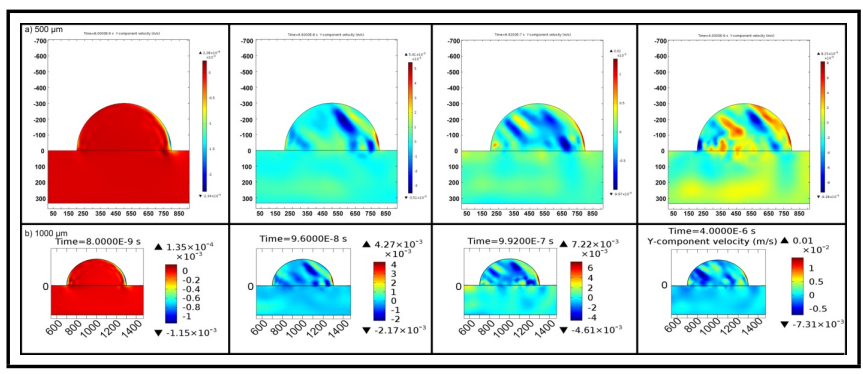

Figure 13. Lateral positions of $500 \mu \mathrm{m} \& 1000 \mu \mathrm{m}$ cut plane results, respectively, in order, row-wise for two cases showing an effective change in results with the passage of time in the simulation.
Table 2. Showing better ranges for the streaming mixing effect produced inside a droplet, in the case of changing droplet size as well as lateral position of the droplet on the surface of the substrate from the centre.

\begin{tabular}{||c|c|}
\hline Parameter & Range of Avg. Velocity Inside Droplet \\
\hline Droplet size & $735 \sim 765$ \\
\hline $\begin{array}{c}\text { Lateral position of droplet } \\
\text { on surface of substrate } \\
\text { from centre }\end{array}$ & $420 \sim 580 \mu \mathrm{m}$ \\
\hline
\end{tabular}

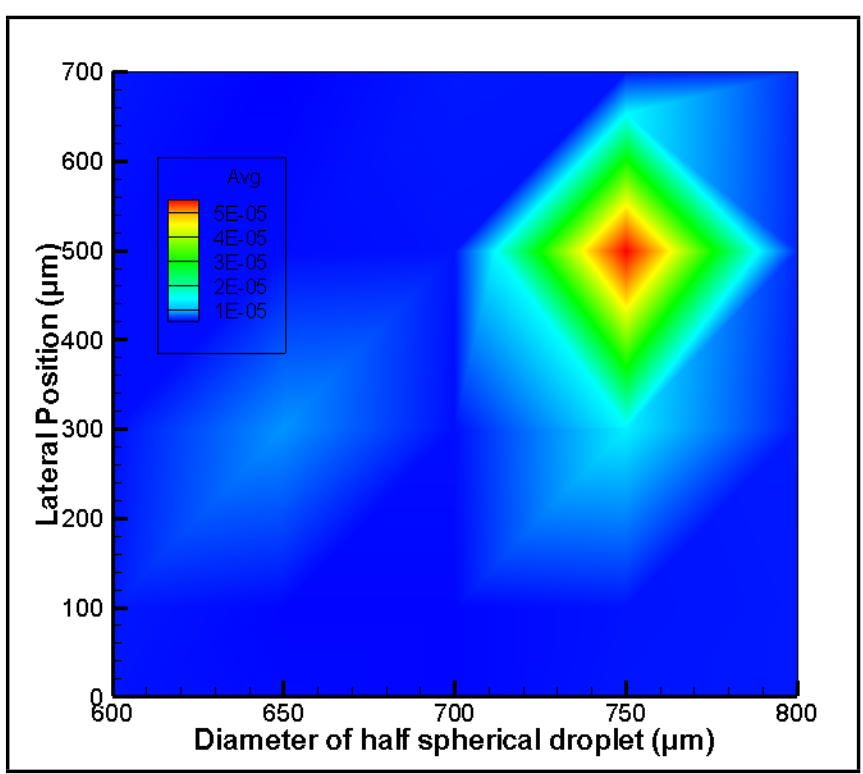

Figure 14. Average velocity contours of the lateral positions and diameter of a half spherical droplet.

for better mixing or streaming produced inside a small droplet. These ranges are presented in Table 2.

\section{CONCLUSIONS}

This study presents the numerical simulations of a SAW to investigate the effect of different parameters like frequency, droplet size, lateral position, input voltage, and lateral position on the velocity inside the droplet. It is observed that a higher input voltage and small diameter of a droplet results in an increase in maximum velocity inside the droplet and the frequency of $20 \mathrm{MHz}$ provides the best streaming or velocity results. The substrate material is also changed and it is concluded that better streaming and velocity values are obtained by keeping lead zirconate titanate and lithium niobate as a substrate material but lithium niobate is the conventional material as it is widely used to simulate the experiments. It is also observed that the streaming and mixing effect is improved when the droplet is kept nearly at the centre of the substrate or exactly at the centre and its effect becomes constant after $70 \mu \mathrm{m}$. It is observed that by changing the lateral position of the droplet and the droplet size (diameter) together, the $350 \mu \mathrm{m}$ droplet at the $300 \mu \mathrm{m}$ lateral position results in the maximum velocity inside the droplet. All these parameters provide valuable information about the droplet lateral position, input voltage, etc., to utilize in different micro-mixing processes hence it can provide vital information for mixing in point-of-care devices.

\section{REFERENCES}

1 Jiao, Z. J., Huang, X. Y., and Nguyen, N. T. Scattering and attenuation of surface acoustic waves in droplet actuation, Journal of Physics A: Mathematical and Theoretical, 
41(35), 355502, (2008). https://dx.doi.org/10.1088/1751$8113 / 41 / 35 / 355502$.

2 Sritharan, K., Strobl, C. J., Schneider, M. F., Wixforth, A., and Guttenberg, Z. V. Acoustic mixing at low Reynold's numbers, Applied Physics Letters, 88(5), 054102, (2006). https://dx.doi.org/10.1063/1.2171482.

3 Alghane, M., Chen, B. X., Fu, Y. Q., Li, Y., Luo, J. K. and Walton, A. J. Experimental and numerical investigation of acoustic streaming excited by using a surface acoustic wave device on a $128^{\circ}$ YX-LiNbO3 substrate, Journal of Micromechanics and Microengineering, 21(1), 015005, (2010). https://dx.doi.org/10.1088/0960$1317 / 21 / 1 / 015005$.

${ }^{4}$ Alghane, M., Fu, Y. Q., Chen, B. X., Li, Y., Desmulliez, M. P. Y., and Walton, A. J. Streaming phenomena in microdroplets induced by Rayleigh surface acoustic wave, Journal of Applied Physics, 109(11), 114901, (2011). https://dx.doi.org/10.1063/1.3586040.

5 Raghavan, R. V., Friend, J. R., and Yeo, L. Y. Particle concentration via acoustically driven microcentrifugation: microPIV flow visualization and numerical modelling studies, Microfluidics and Nanofluidics, 8(1), 73, (2010). https://dx.doi.org/10.1007/s10404-009-0452-3.

${ }^{6}$ Liu, G., Chai, C., and Yao, B. Rapid evaluation of Salmonella pullorum contamination in chicken based on a portable amperometric sensor, Biosens. Bioelectron, 4(137), 137-143, (2013).

7 Travagliati, M., Shilton, R. J., Pagliazzi, M., Tonazzini, I., Beltram, F., and Cecchini, M. Acoustofluidics and whole-blood manipulation in surface acoustic wave counterflow devices, Analytical chemistry, 86(21), 1063310638, (2014). https://dx.doi.org/10.1021/ac502465s.

${ }^{8}$ Du, X. Y., Fu, Y. Q., Luo, J. K., Flewitt, A. J., and Milne, W. I. Microfluidic pumps employing surface acoustic waves generated in $\mathrm{ZnO}$ thin films, Journal of Applied Physics, 105(2), 024508, (2009). https://dx.doi.org/10.1063/1.3068326.

9 Brunet, P., Baudoin, M., Matar, O. B., and Zoueshtiagh, F. Droplet displacements and oscillations induced by ultrasonic surface acoustic waves: a quantitative study, Physical Review E, 81(3), 036315, (2010). https://dx.doi.org/10.1103/PhysRevE.81.036315.

10 Alghane, M., Fu, Y. Q., Chen, B. X., Li, Y., Desmulliez, M. P. Y., and Walton, A. J., 2012. Frequency effect on streaming phenomenon induced by Rayleigh surface acoustic wave in microdroplets, Journal of Applied Physics, 112(8), 084902, (2012). https://dx.doi.org/10.1063/1.4758282.

11 Rogers, P. R., Friend, J. R., and Yeo, L. Y. Exploitation of surface acoustic waves to drive size-dependent microparticle concentration within a droplet, Lab on a Chip, 10(21), 2979-2985, (2010). https://dx.doi.org/10.1039/C004822D.

12 Daniel, S., Chaudhury, M. K., and De Gennes, P. G. Vibration-actuated drop motion on surfaces for batch microfluidic processes, Langmuir, 21(9), 4240-4248, (2005). https://dx.doi.org/10.1021/la046886s.
${ }^{13}$ Newton, M. I., Banerjee, M. K., Starke, T. K. H., Rowan, S. M., and McHale, G. Surface acoustic waveliquid drop interactions, Sensors and Actuators A: Physical, 76(1-3), 89-92, (1999). https://dx.doi.org/10.1016/S09244247(98)00356-2.

${ }^{14}$ Qi, A., Friend, J. R., Yeo, L. Y., Morton, D. A., McIntosh, M. P., and Spiccia, L. Miniature inhalation therapy platform using surface acoustic wave microfluidic atomization, Lab on a Chip, 9(15), 2184-2193, (2009). https://dx.doi.org/10.1039/B903575C.

15 Franke, T., Abate, A. R., Weitz, D. A., and Wixforth, A. Surface acoustic wave (SAW) directed droplet flow in microfluidics for PDMS devices, Lab on a Chip, 9(18), 26252627, (2009). https://dx.doi.org/10.1039/B906819H.

${ }^{16}$ Frommelt, T., Gogel, D., Kostur, M., Talkner, P., Hanggi, P., and Wixforth, A. Flow patterns and transport in Rayleigh surface acoustic wave streaming: Combined finite element method and raytracing numerics versus experiments, IEEE transactions on ultrasonics, ferroelectrics, and frequency control, 55(10), 2298-2305, (2008). https://dx.doi.org/10.1109/TUFFC.928.

17 Luo, J. K., Fu, Y. Q., Li, Y., Du, X. Y., Flewitt, A. J., Walton, A. J., and Milne, W. I. Moving-part-free microfluidic systems for lab-on-a-chip, Journal of Micromechanics and Microengineering, 19(5), 054001, (2009). https://dx.doi.org/10.1088/0960-1317/19/5/054001.

18 Jung, J. H., Destgeer, G., Ha, B., Park, J., and Sung, H. J. On-demand droplet splitting using surface acoustic waves, Lab on a Chip, 16(17), 3235-3243, (2016). https://dx.doi.org/10.1039/C6LC00648E.

${ }_{19}$ Ding, X., Li, P., Lin, S. C. S., Stratton, Z. S., Nama, N., Guo, F., Slotcavage, D., Mao, X., Shi, J., Costanzo, F., and Huang, T. J. Surface acoustic wave microfluidics, Lab on a Chip, 13(18), 3626-3649, (2013). https://dx.doi.org/10.1039/C3LC50361E.

${ }^{20}$ Renaudin, A., Tabourier, P., Zhang, V., Camart, J. C., and Druon, C. SAW nanopump for handling droplets in view of biological applications, Sensors and Actuators B: Chemical, 113(1), 389-397, (2006). https://dx.doi.org/10.1016/j.snb.2005.03.100.

${ }^{21}$ Imai, T., Nakahata, H., and Fujimori, N. Sumitomo Electric Industries Ltd, Surface acoustic wave device. U.S. Patent 4,952,832, (1990).

22 Zhang, A. L., Wu, Z. Q., and Xia, X. H. Transportation and mixing of droplets by surface acoustic wave, Talanta, 84(2), 293-297, (2011). https://dx.doi.org/10.1016/j.talanta.2011.01.017.

23 Alghane, M. M. Surface acoustic wave streaming in a microfluidic system, doctoral dissertation, Heriot-Watt University, 30-31, (2013).

${ }^{24}$ Mohammadi, M. M. A comparison between quartz and PZT ceramic for sensoric applications, Res. Desk, 2, 321-325, (2013).

${ }^{25}$ Li, D., Zhang, S., Yang, W., and Zhang, W. 2014. Corrosion monitoring and evaluation of reinforced concrete structures utilizing the ultrasonic guided wave technique, International Journal of Distributed Sensor Networks, 10(2), 827130, (2014).https://dx.doi.org/10.1155/2014/827130. 